\title{
CrystEngComm
}

Check for updates

Cite this: CrystEngComm, 2017, 19, 5412

Received 31st July 2017

Accepted 4th August 2017

DOI: $10.1039 / c 7 c e 01396 e$

rsc.li/crystengcomm

\section{Facile synthesis of scandium fluoride oriented single-crystalline rods and urchin-like structures by a gas-solution interface technique $\uparrow$}

\author{
L. B. Gulina, (D)* V. P. Tolstoy, (D) I. A. Kasatkin (iD and I. V. Murin (D)
}

A facile and effective method to obtain urchin-like structures and ordered arrays of rod-like crystals of scandium fluoride by an interfacial interaction between solution and gaseous reagents (Gas-Solution Interface Technique, GSIT) is reported. The rod crystals with tetragonal crystal lattice can be as thin as ca. 200 $\mathrm{nm}$ and as long as ca. $7 \mu \mathrm{m}$. The effect of solution concentration and reaction time on the morphology of the product is reported. The synthesized crystals were characterized by SEM, TEM, HRTEM, XRD analyses, EDS, XPS, and FT-IR spectroscopy. A hypothesis is proposed on the formation of rod crystals during the GSIT process.

\section{Introduction}

Currently, interface-mediated synthetic approaches, in which the interface determines the transport behaviour of ions and molecules, are widely applied to controlled crystal growth and fabrication of nano- or microstructures. ${ }^{1}$ In particular, the air-water interface is used for characterization of surfactant molecules and for obtaining Langmuir films. ${ }^{2}$ Self-assembly of building blocks such as molecules, ions, particles, micelles, etc. at the surface of water is the main driving force of Langmuir layer formation. Usually, surface-active molecules assist in this process. For example, mineralization with the assistance of an amphiphilic block copolymer ${ }^{3}$ and silk fibroinmediated biomineralization ${ }^{4}$ were carried out at the air-water interface. Alternatively, isothermal evaporation can be applied to fabrication of organic crystals by a drop-casting method at the air-liquid interface. ${ }^{5}$ In this way, the solvent polarity is used to control the morphology of the product. Hydrolysis and decomposition of a complex compound have been used ${ }^{6}$ to obtain a self-assembled film formed from oriented inorganic crystals at the air-liquid interface. In the same way, the aminolytic reaction in solution along with the self-assembly process allowed the formation of three-dimensional hierarchical inorganic architectures. ${ }^{7}$

Recently, a facile gas-solution interface technique (GSIT) was successfully applied to synthesize well-known inorganic materials in the form of thin films or microtubes ${ }^{8-11}$ at room temperature under soft chemistry conditions without surfac-

Saint Petersburg State University, 7/9 Universitetskaya nab, St. Petersburg, 199034, Russia. E-mail: l.gulina@spbu.ru; Fax: +7(812)428 6859;

Tel: $+7(812) 4284104$

$\dagger$ Electronic supplementary information (ESI) available: Additional SEM images and XRD patterns. See DOI: 10.1039/c7ce01396e tants. The interaction between a gaseous reagent and dissolved cations at the surface of solution plays a key role in this type of synthesis. The opportunity to obtain inorganic fluorides by GSIT was first demonstrated in ref. 12 and 13 with the example of $2 \mathrm{D}$ oriented strained $\mathrm{LaF}_{3}$ crystals with high ionic mobility. The effect of solution doping on modifying the properties of lanthanum fluoride was studied. ${ }^{14-16}$

In this work, we applied GSIT to synthesize rod-like crystals of scandium fluoride $\left(\mathrm{ScF}_{3}\right) . \mathrm{ScF}_{3}$ is an important component of materials with ionic conductivity. ${ }^{17,18}$ Doped $\mathrm{ScF}_{3}$ demonstrates up/down-conversion fluorescence properties, ${ }^{19}$ and can be applied to bioimaging. ${ }^{20}$ Importantly, scandium fluoride belongs to a very specific and narrow group of materials demonstrating negative thermal expansion (NTE) in the broad temperature range from $10 \mathrm{~K}$ to $1100 \mathrm{~K}^{21-23}$ It is known that the magnitude of NTE is reduced with decreasing crystal size. ${ }^{24}$ Crystal morphology is also known to affect the optical, mechanical, electrochemical, catalytic and other properties of materials. ${ }^{25-28}$ Tuning of NTE in crystals by tuning the phase composition and crystal morphology allows designing new materials with controllable thermal properties. We focus here on demonstrating the potential of the GSIT method to synthesize $\mathrm{ScF}_{3}$ rod-like crystals. In our future work, we are planning to optimize the synthesis strategy and perform deeper and detailed characterization of the phases obtained.

\section{Results and discussion}

As a result of the interaction between a $0.01 \mathrm{M}$ solution of scandium chloride and gaseous HF, separated microclusters of solid particles are formed on the solution surface, which are not connected to each other. When a $0.02 \mathrm{M} \mathrm{ScCl}_{3}$ 
solution is used as a reagent, a fractal network of solid particles is formed on the surface (Fig. 1a). After washing and transferring onto a silicon wafer, the structures were characterized by SEM (Fig. 1b-f). As seen in the images, the synthesized network consists of urchin-like structures formed from rods with lengths up to 6-7 micrometers. The rod length depends on the treatment time. During the first $30 \mathrm{~min}$ of reaction, the crystals grew up to a size of $3-4$ micrometers (Fig. 1c). After $60 \mathrm{~min}$, the maximum length was 6-7 micrometers (Fig. 1d). It is seen that several rod-like crystals grew from a single seed. In some photographs, one can also see crystals with a cubic morphology (Fig. 1e) up to $500 \mathrm{~nm}$ in size. In some images of the largest rod-like crystals, faceting characteristics can be observed (Fig. 1f).

A typical X-ray diffraction (XRD) pattern of the urchin-like structures is shown in Fig. 2. A couple of high-intensity diffraction peaks at the Bragg angles $2 \theta=22.1$ and $2 \theta=45.2$ degrees and other peaks are assigned to the crystal planes of $\mathrm{ScF}_{3}$ with a cubic crystal lattice and the $P m \overline{3} m$ space group [ICDD PDF \#00-046-1243] showing a strong [100] texture. The additional series of diffraction peaks marked by blue color and asterisk in the figure can be assigned to the crystal planes of tetragonal scandium fluoride [ICDD PDF \#00-0500238]. We carried out additional GSIT synthesis of scandium fluorides from $\mathrm{ScCl}_{3}$ and $\mathrm{Sc}\left(\mathrm{NO}_{3}\right)_{3}$ solutions with $\mathrm{pH}=1$ and $\mathrm{pH}=3$. In each case, the XRD patterns were identical to that presented in Fig. 2.

The TEM images of the urchin-like structures are shown in Fig. 3. As seen in Fig. 3a-c, the structure synthesised over 20 min consists of rods with lengths up to 2 micrometers.

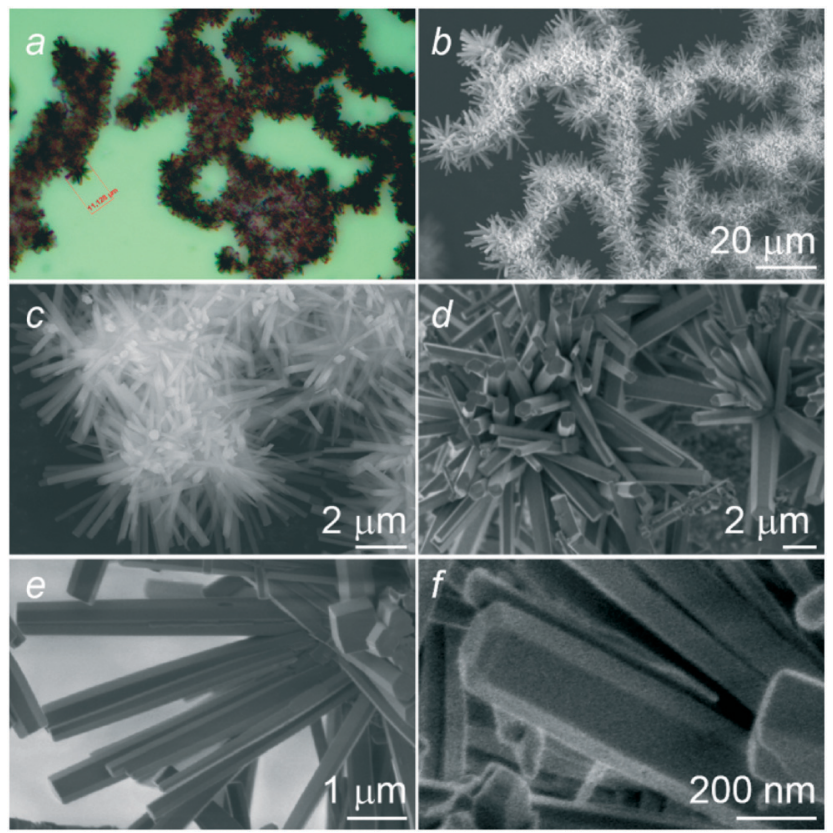

Fig. 1 Images of urchin-like structures synthesized from $0.02 \mathrm{M} \mathrm{ScCl}_{3}$ solution: a) optical reflection image; $b$ and c) SEM images of structures synthesized over 30 min with different magnifications; d-f) SEM images of structures synthesized over $60 \mathrm{~min}$ with different magnifications.

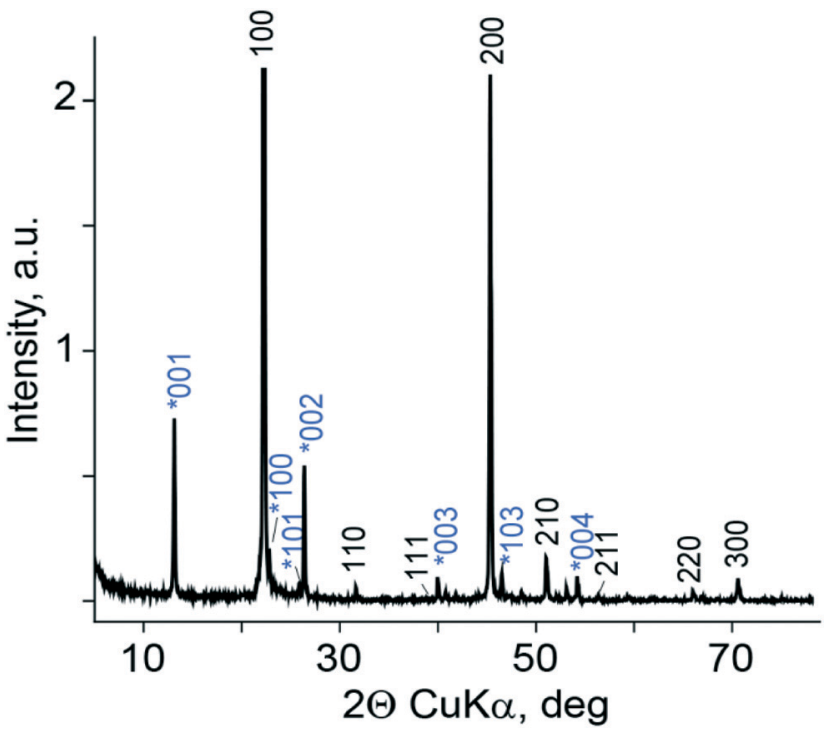

Fig. 2 X-ray diffraction pattern of scandium fluoride crystals.

The SAED patterns (insets in Fig. $2 \mathrm{~d}$ and e) agree with the XRD results and show a tetragonal lattice with parameters close to those reported in ref. 29 for $\mathrm{ScF}_{2.76}: a=0.379 \mathrm{~nm}$ and $c=0.674 \mathrm{~nm}$. The crystals are elongated along the [001] direction.

When a more concentrated $0.1 \mathrm{M}$ scandium salt solution was used as a precursor, a continuous film was formed. The surface of the film consisted of cubic crystals (Fig. 4a and c) on the atmosphere side, but the oriented rod crystals grew on the opposite side facing the solution (Fig. 4b). Fig. S1† shows SEM images of the non-continuous film grown on the surface of a $0.05 \mathrm{M}$ solution. The islands in which the film is continuous have diameters up to $20 \mu \mathrm{m}$, and the rod-like crystals in the central parts are preferably oriented with their long axes along the normal direction to the film surface. The ordering is disturbed on the edges of these areas, where urchin-like rims are formed. The diameters of the islands and the overall degree of the solution surface coverage by the film can be adjusted by changing the solution concentration within the range of 0.02-0.1 $\mathrm{M}$.

The elemental compositions of the synthesized structures were determined with EDS (Fig. 5). A typical EDS spectrum is shown in Fig. 5b; only Sc and F were detected. The resulting average ratio of $\mathrm{Sc} / \mathrm{F}$ is $23 / 77$ which corresponds to stoichiometric $\mathrm{ScF}_{3}$.

The X-ray photoelectron spectroscopy (XPS) results are plotted in Fig. 6. The binding energies of Sc $\left(2 \mathrm{p}_{1 / 2}, 410.3 \mathrm{eV}\right.$ and $\left.2 \mathrm{p}_{3 / 2}, 406.0 \mathrm{eV}\right)$ and $\mathrm{F}(1 \mathrm{~s}, 686.3 \mathrm{eV})$ can be readily observed in the spectra (Fig. $6 \mathrm{~b}$ and c). The Sc $2 \mathrm{p}$ peak has significantly split spin-orbit components $(\Delta=4.3 \mathrm{eV})$, which correspond to $\mathrm{Sc}^{3+} .^{30}$ The $\mathrm{F} 1 \mathrm{~s}$ peak centered at $686.3 \mathrm{eV}$ may be attributed to the metal-fluorine bonds. ${ }^{31}$ According to the XPS analysis, the Sc/F ratio is close to 24/76 which conforms to the nominal composition of $\mathrm{ScF}_{3}$.

The results of FT-IR spectroscopy investigation (Fig. 7) agree with these data. The absorption bands in the 600-480 


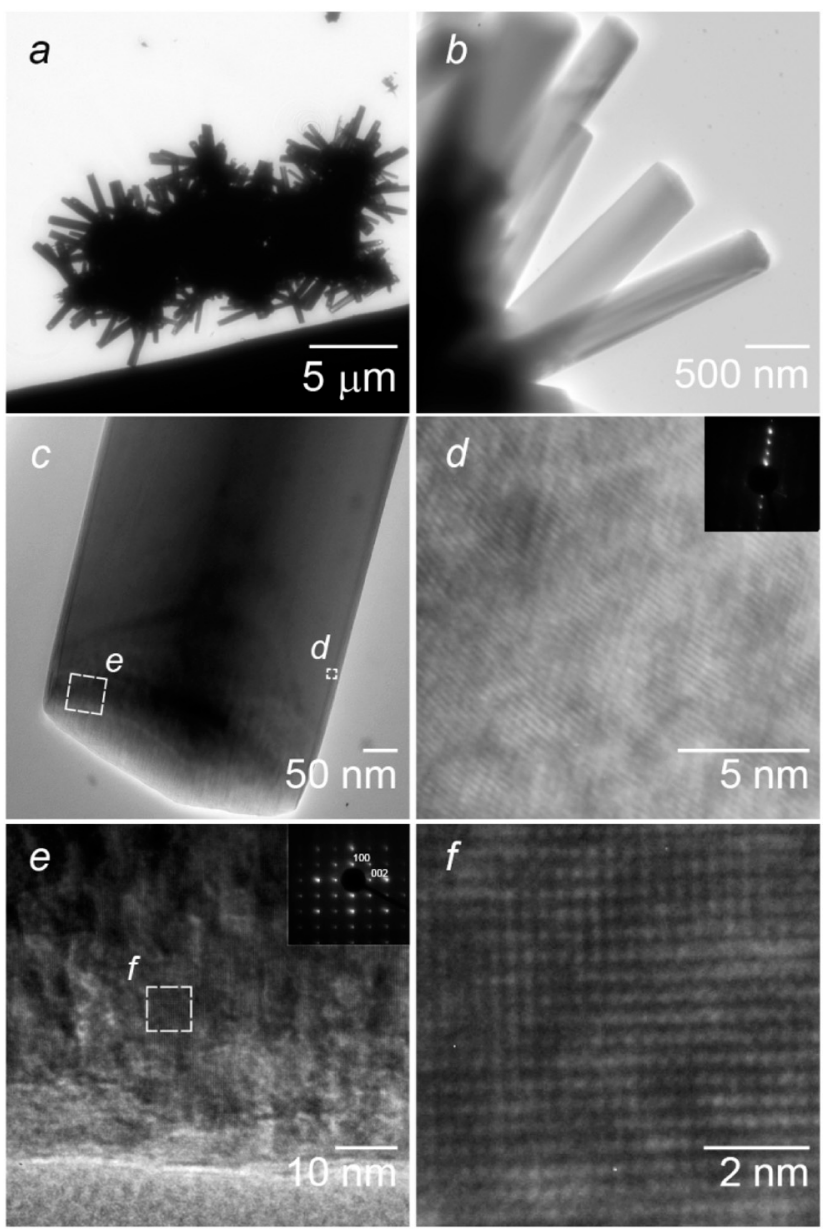

Fig. 3 TEM and HRTEM images of scandium fluoride crystals synthesized over 20 min of reaction: $a$ and b) overview images of the urchin-like structure with different magnifications; c) single rod crystal; the marked area is magnified in $d$ and e; d) HRTEM image of the side edge of the rod crystal with the corresponding SAED pattern in the inset; e) HRTEM image of the top edge of the rod crystal with the SAED pattern in the inset; f) magnified HRTEM image of the marked area in e.

$\mathrm{cm}^{-1}$ region can be related to the Sc-F vibration, particularly the band at $605 \mathrm{~cm}^{-1}$ corresponding to the $v_{1}\left(a_{1}^{\prime}\right)$ fundamental frequency in $\mathrm{ScF}_{3}$ (ref. 32-34) and the two components, $495 \mathrm{~cm}^{-1}(s)$ and $550 \mathrm{~cm}^{-1}(v)$, associated with the rotation of the octahedral $\left[\mathrm{ScF}_{6}\right]$ group. ${ }^{35,36}$ The absorption bands with maxima at $3380 \mathrm{~cm}^{-1}$ and $3230 \mathrm{~cm}^{-1}$ in this spectrum should be attributed, respectively, to the valence oscillations of the $\mathrm{O}-\mathrm{H}$ bonds of the water molecules ${ }^{37}$ and the HF stretching bands of the hydrogen fluoride species. ${ }^{38,39}$

The following model of formation of oriented $\mathrm{ScF}_{3}$ crystals may be suggested. In the initial time of interaction, cubic crystals are formed on the solution surface, and they acted as parent crystals or nucleation centers of rods. When the concentration of the scandium salt in solution was low, the cubes floated on the solution surface freely and separately from each other, and growth of tetragonal crystals occurred in a disordered manner in all directions. In the case of more concentrated solutions, the surface was completely covered

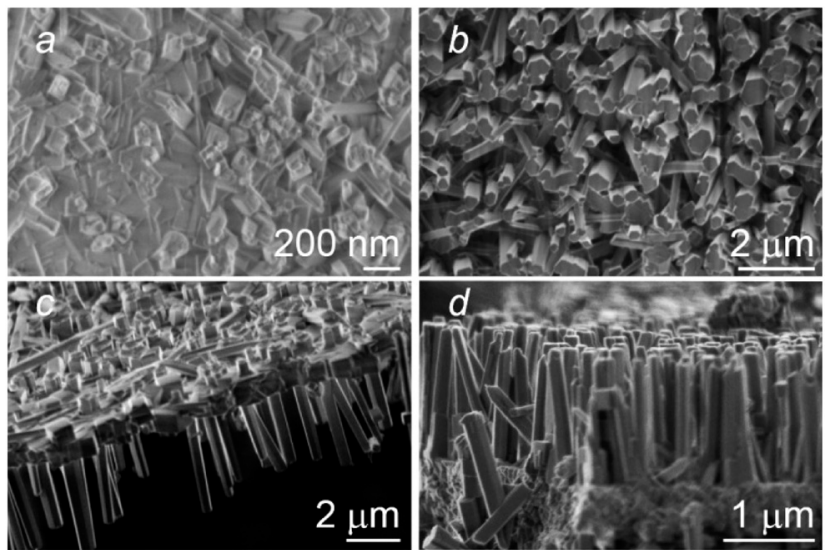

Fig. 4 SEM images of scandium fluoride rod crystals synthesized from $0.1 \mathrm{M} \mathrm{ScCl}_{3}$ solution: a) top view image; b) bottom view image; $c$ and d) cross-sectional views under different angles and with different magnifications.

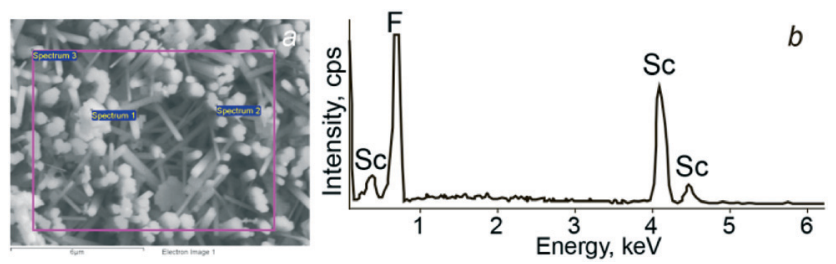

Fig. 5 EDS analysis of scandium fluoride crystals: a) SEM image showing the points of analyses; b) EDS spectrum of area 3.

with oriented cubic crystals and the growth of oriented rods was observed in the direction orthogonal to the gas-solution interface towards the liquid phase. The uniform preferred orientation of the rods which is clearly seen in Fig. 4 can be easily explained by the evolutionary mechanism of geometric selection: among the randomly nucleated crystals of the tetragonal phase, only those that had chances to grow further nucleated in the preferred orientation.

To support the suggested model, we can mention the fact that the cubic phase concentration detectable with XRD is lower in urchin-like clusters than that in complete films (Fig. S2 $\dagger$ ). In the SEM images, it is clearly seen that the cubes in the clusters are hidden in the centers of the "urchins" (Fig. S3†) and only rarely appear outside them (Fig. 1e, upper right corner, for instance). Since the rods grow only on one side of the complete films, the concentration of

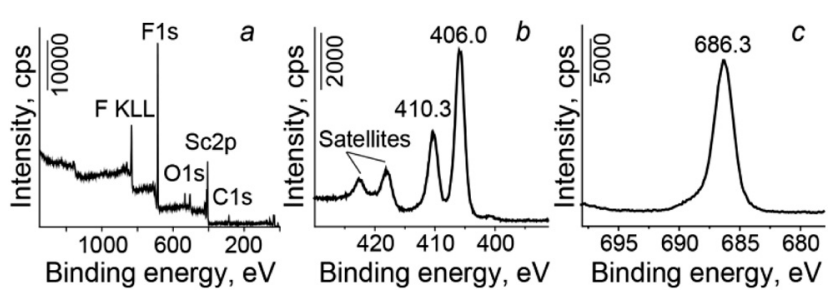

Fig. 6 The XPS spectra of scandium fluoride crystals: a) general spectrum, b) Sc 2p and c) F 1s XPS spectra. 


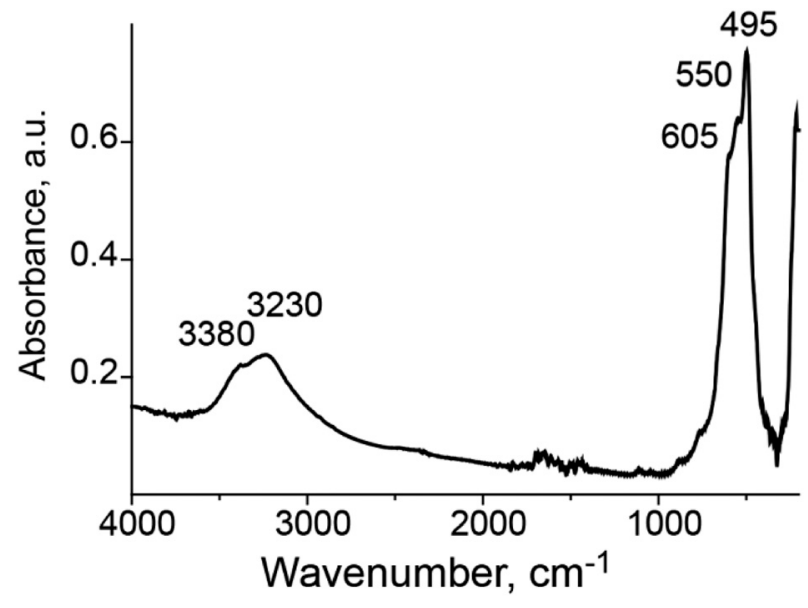

Fig. 7 FT-IR spectrum of scandium fluoride crystals.

the cubic phase is higher. On the other hand, only short rods of 1.0-1.4 micrometers in length and a large quantity of cubic crystals are observed in the initial step (10 $\mathrm{min})$ of synthesis from a highly concentrated solution (Fig. S4†). This demonstrates that the cubic phase crystallizes first and provides the centers of heterogeneous nucleation of the rods.

\section{Experimental}

\section{Chemicals and materials}

$\mathrm{Sc}_{2} \mathrm{O}_{3}$ (extra pure) and a concentrated solution of $40 \% \mathrm{HF}$ (extra pure) provided by Vekton were taken as the reagents. All aqueous solutions were prepared using Milli-Q high-purity water with a resistivity more than $18 \mathrm{M} \Omega \mathrm{cm}^{-1}$. The aqueous solutions of the corresponding $\mathrm{ScCl}_{3}$ or $\mathrm{Sc}\left(\mathrm{NO}_{3}\right)_{3}$ were obtained by dissolving the stoichiometric oxide in dilute $\mathrm{HCl}$ or $\mathrm{HNO}_{3}$ solution. The concentration of the scandium salt solutions used for the synthesis varied in the range of 0.01-0.10 $\mathrm{M}$ and their $\mathrm{pH}$ values in the range of 1.0-3.0.

Single-crystal silicon wafers served as the substrates in performing the analysis of the synthesized crystals. The substrates were treated in "piranha" solution, i.e. a mixture of $30 \% \mathrm{H}_{2} \mathrm{O}_{2}$ and concentrated $\mathrm{H}_{2} \mathrm{SO}_{4}$ (3:7 by volume), for 10 min and then rinsed thoroughly in water.

\section{Synthetic procedures}

The synthesis of the $\mathrm{ScF}_{3}$ crystals by GSIT was carried out at room temperature in a Teflon hermetically sealed reactor similar to that described in ref. 40 for $\mathrm{LaF}_{3}$ nanocrystals. Briefly, the surface of a water solution of the scandium salt was brought into contact with a HF-containing atmosphere in the reactor. The processing time varied from 10 to $60 \mathrm{~min}$.

The experiments have shown that a transparent colorless film was formed on the surface of the scandium salt solution during the HF treatment. After that, the film was transferred to the surface of pure distilled water in order to remove the excess solution and left there for $10 \mathrm{~min}$ of exposure. This procedure was repeated twice. Next, the film was transferred to a silicon substrate surface, dried at room temperature and analysed by optical microscopy, X-ray diffraction, SEM, EDS, FT-IR spectroscopy and XPS. The urchin-like structures were investigated as-synthesized on silicon; the continuous film with oriented rods was fixed on the support with carbon conductivity tape for SEM and XPS analyses. For the TEM and HRTEM investigation, the as-synthesized structures were transferred onto an $\mathrm{Au}$ grid coated with an amorphous carbon supporting film.

\section{Characterization}

X-Ray powder diffraction analysis was performed on a Rigaku "Miniflex II" diffractometer using $\mathrm{CuK} \alpha$ radiation under 30 $\mathrm{kV}$ voltage. The morphologies of the samples were determined using an optical microscope (Carl-Zeiss Axio Scope A1) and a scanning electron microscope (Zeiss Merlin) at an accelerating voltage of $2 \mathrm{kV}$. The chemical composition of the samples was defined by EPMA using a SEM equipped with an INCAx-act Energy EDX analyzer (Oxford Instruments). TEM and HRTEM investigations were performed with a Zeiss Libra 200 FE TEM. XPS analysis of the surface was conducted with a Thermo Fisher Scientific ESCAlab 250Xi. Infrared spectra were obtained on a Bruker Vertex 70 infrared spectrometer.

\section{Conclusions}

In this study, large-area free-standing rods and urchin-like structures of scandium fluoride have been prepared successfully in the absence of catalysts or templates under soft chemistry conditions.

$\mathrm{ScF}_{3}$ rods can be synthesized by interfacial growth from the solution and gaseous reagents. The as-prepared $\mathrm{ScF}_{3}$ samples exhibit a mixed phase composition consisting of cubic and tetragonal crystals. The composition of the samples corresponds to scandium trifluoride with a possible excess of fluorine originating from HF occlusion. The morphology of the product depends on the concentration of solution and the reaction time. The longest $\mathrm{ScF}_{3}$ rods were over 7 micrometers long.

Non-cubic crystals will most probably exhibit anisotropic thermal expansion, the property which can be utilized when designing new materials with controllable thermal expansion (CTE). In our future work, we are planning to perform a detailed study on the thermal behavior of this phase.

\section{Conflicts of interest}

There are no conflicts to declare.

\section{Acknowledgements}

This work was supported by the Russian Science Foundation (project no. 16-13-10223). We thank the following Resource Centers of Saint Petersburg State University for their technical assistance with the characterization of the synthesized product: Centre for X-ray Diffraction Studies, Centre for Physical 
Methods of Surface Investigation, Thermogravimetric and Calorimetric Research Centre, and Interdisciplinary Resource Centre for Nanotechnology.

\section{References}

1 X. Wang, Q. Peng and Y. Li, Acc. Chem. Res., 2007, 40, 635-643.

2 J. Y. Park and R. C. Advincula, Soft Matter, 2011, 7, 9829-9843.

3 D. Hentrich, M. Junginger, M. Bruns, H. G. Borner, J. Brandt, G. Brezesinski and A. Taubert, CrystEngComm, 2015, 17, 6901-6913.

4 W. Hao, D. Porter, X. Wang and Z. Shao, CrystEngComm, 2014, 16, 9176-9184.

5 H. Shang, H. Wang, N. Gao, F. Shen, X. Li and Y. Ma, CrystEngComm, 2012, 14, 869-874.

6 Y. Masuda and K. Kato, Thin Solid Films, 2013, 544, 567-570.

7 M. V. Vaishampayan, I. S. Mulla and S. S. Joshi, Langmuir, 2011, 27, 12751-12759.

8 V. P. Tolstoy and L. B. Gulina, J. Nano- Electron. Phys., 2013, 5, 01003.

9 V. P. Tolstoi and L. B. Gulina, Russ. J. Gen. Chem., 2013, 83, 1635-1639.

10 V. P. Tolstoy and L. B. Gulina, Langmuir, 2014, 30, 8366-8372.

11 L. B. Gulina, V. P. Tolstoy and E. V. Tolstobrov, Mendeleev Commun., 2017, 27, accepted in press.

12 L. B. Gulina, M. Schäfer, A. F. Privalov, V. P. Tolstoy and I. V. Murin, J. Chem. Phys., 2015, 143, 234702.

13 L. B. Gulina, V. P. Tolstoy, I. A. Kasatkin and Y. V. Petrov, J. Fluorine Chem., 2015, 180, 117-121.

14 L. B. Gulina, M. Schäfer, A. F. Privalov, V. P. Tolstoy, I. V. Murin and M. Vogel, J. Fluorine Chem., 2016, 188, 185-190.

15 L. B. Gulina, V. P. Tolstoy, A. A. Lobinskiy and Y. V. Petrov, Russ. J. Gen. Chem., 2016, 86, 2057-2060.

16 L. B. Gulina, V. P. Tolstoy, I. A. Kasatkin, I. E. Kolesnikov and D. V. Danilov, J. Fluorine Chem., 2017, 200, 18-23.

17 P. P. Fedorov, V. Trnovcova, G. I. Kocherba and B. P. Sobolev, Crystallogr. Rep., 1995, 40, 663-667.

18 V. Trnovcová, P. P. Fedorov, I. I. Buchinskaya, V. Šmatko and F. Hanic, Solid State Ionics, 1999, 119, 181-189.

19 J. Cao, L. Yuan, S. Hu, J. Tang, X. Zhou and J. Yang, CrystEngComm, 2016, 18, 5940-5951.

20 Y. Ai, D. Tu, W. Zheng, Y. Liu, J. Kong, P. Hu, Z. Chen, M. Huang and X. Chen, Nanoscale, 2013, 5, 6430-6438.
21 S. Piskunov, P. A. Žguns, D. Bocharov, A. Kuzmin, J. Purans, A. Kalinko, R. A. Evarestov, S. E. Ali and F. Rocca, Phys. Rev. $B, 2016,93,214102$.

22 N. I. Sorokin, D. N. Karimov, V. V. Grebenev and B. P. Sobolev, Crystallogr. Rep., 2016, 61, 270-274.

23 L. Hu, J. Chen, A. Sanson, H. Wu, C. Guglieri Rodriguez, L. Olivi, Y. Ren, L. Fan, J. Deng and X. Xing, J. Am. Chem. Soc., 2016, 138, 8320-8323.

24 C. Yang, P. Tong, J. C. Lin, X. G. Guo, K. Zhang, M. Wang, Y. Wu, S. Lin, P. C. Huang, W. Xu, W. H. Song and Y. P. Sun, Appl. Phys. Lett., 2016, 109, 023110.

25 F. C. Meldrum and H. Cölfen, Chem. Rev., 2008, 108, 4332-4432.

26 L. Bahrig, S. G. Hickey and A. Eychmuller, CrystEngComm, 2014, 16, 9408-9424.

27 M. Ding, C. Lu, Y. Song, Y. Ni and Z. Xu, CrystEngComm, 2014, 16, 1163-1173.

28 K. M. Ryan, S. Singh, P. Liu and A. Singh, CrystEngComm, 2014, 16, 9446-9454.

29 P. Melnikov, M. Nalin and Y. Messaddeq, J. Alloys Compd., 1997, 262-263, 296-298.

30 M. C. Biesinger, L. W. M. Lau, A. R. Gerson and R. S. C. Smart, Appl. Surf. Sci., 2010, 257, 887-898.

31 J. F. Moulder, W. F. Stickle, P. E. Sobol and K. D. Bomben, Handbook of X-ray Photoelectron Spectroscopy, Physical Electronics Inc., Minnesota, 1995.

32 J. W. Hastie, R. H. Hauge and J. L. Margrave, J. Less-Common Met., 1975, 39, 309-334.

33 V. G. Solomonik and A. A. Mukhanov, J. Struct. Chem., 2012, 53, 28-34.

34 V. G. Solomonik, J. F. Stanton and J. E. Boggs, J. Chem. Phys., 2005, 122, 094322.

35 P. Melnikov and L. N. Komissarova, J. Phys. Chem. Solids, 2006, 67, 1899-1900.

36 A. S. Krylov, M. S. Molokeev, S. V. Misyul, S. N. Krylova, A. S. Oreshonkov, A. A. Ivanenko, V. A. Zykova, Y. N. Ivanov, A. A. Sukhovsky, V. N. Voronov, I. N. Safonov and A. N. Vtyurin, CrystEngComm, 2016, 18, 8472-8486.

37 V. P. Tolstoy, I. A. Chernyshova and V. A. Scryshevsky, Handbook of infrared spectroscopy of ultrathin films, WileyInterscience, New Jersey, 2003.

38 V. P. Tolstoi, B. S. Zhuchkov and I. V. Murin, Inorg. Mater., 2000, 36, 86-87.

39 R. L. Redington and D. F. Hamill, J. Chem. Phys., 1983, 80, 2446-2461.

40 L. B. Gulina and V. P. Tolstoy, Russ. J. Gen. Chem., 2014, 84, 1472-1475. 